\title{
Ultrafast Low-Temperature Crystallization of Solar Cell Graded Formamidinium-Cesium Mixed-Cation Lead Mixed-Halide Perovskites Using a Reproducible Microwave-Based Process
}

\author{
Maria João Brites, ${ }^{* \dagger}{ }^{\dagger}$ Maria Alexandra Barreiros, ${ }^{\dagger}$ Victoria Corregidor, ${ }^{\ddagger}$ Luis C. Alves, ${ }^{\S}$

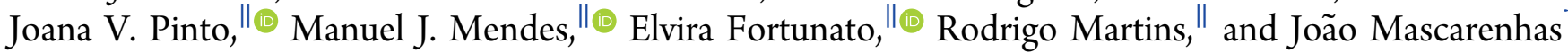 \\ ${ }^{\dagger}$ Laboratório Nacional de Energia e Geologia, LNEG/UER, Estrada do Paço do Lumiar, 22, 1649-038 Lisboa, Portugal \\ ${ }^{\ddagger}$ IPFN, Campus Tecnológico e Nuclear, Instituto Superior Técnico and ${ }^{\S} \mathrm{C} 2 \mathrm{TN}$, Campus Tecnológico e Nuclear, Instituto Superior \\ Técnico, Universidade de Lisboa, E. N.10, 2695-066 Bobadela LRS, Portugal \\ "CENIMAT-I3N, Faculdade de Ciências e Tecnologia, Universidade Nova de Lisboa, Campus de Caparica, 2829-516 Caparica, \\ Portugal
}

\section{Supporting Information}

ABSTRACT: The control of morphology and crystallinity of solution-processed perovskite thin-films for solar cells is the key for further enhancement of the devices' power conversion efficiency and stability. Improving crystallinity and increasing grain size of perovskite films is a proven way to boost the devices' performance and operational robustness, nevertheless this has only been achieved with high-temperature processes. Here, we present an unprecedented low-temperature $\left(<80^{\circ} \mathrm{C}\right)$ and ultrafast microwave (MW) annealing process to yield uniform, compact, and crystalline $\mathrm{FA}_{0.83} \mathrm{Cs}_{0.17} \mathrm{~Pb}\left(\mathrm{I}_{(1-x)} \mathrm{Br}_{x}\right)_{3}$ perovskite films with full coverage and micrometer-scale grains. We demonstrate that the nominal composition

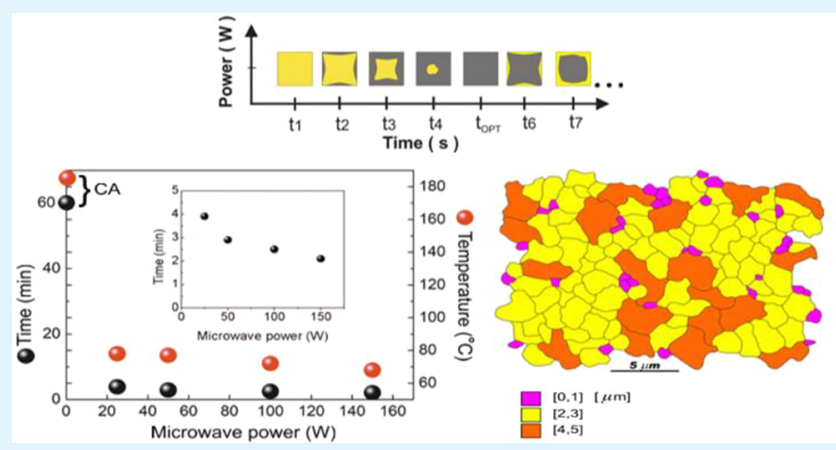
$\mathrm{FA}_{0.83} \mathrm{Cs}_{0.17} \mathrm{PbI}_{1.8} \mathrm{Br}_{1.2}$ perovskite films annealed at $100 \mathrm{~W} \mathrm{MW}$ power present the same band gap, similar morphology, and crystallinity of conventionally annealed films, with the advantage of being produced at a lower temperature (below $80{ }^{\circ} \mathrm{C}$ vs 185 ${ }^{\circ} \mathrm{C}$ ) and during a very short period of time $(\sim 2.5 \mathrm{~min}$ versus $60 \mathrm{~min})$. These results open new avenues to fabricate band gap tunable perovskite films at low temperatures, which is of utmost importance for mechanically flexible perovskite cells and monolithic perovskite based tandem cells applications.

KEYWORDS: microwave annealing, fast low-temperature processing, micrometer-scale grains, mixed-cation lead mixed-halide perovskites, perovskite chemical composition

\section{INTRODUCTION}

Organo-metal halide perovskites have emerged as an extremely promising photovoltaic (PV) technology due to high power conversion efficiencies (PCE) and low fabrication cost. In a span of a few years, the record single-junction efficiency of perovskite solar cells has reached $22.7 \%^{1}$ making it the most efficient solution-processed PV technology. Furthermore, their wide tunable band gap ${ }^{2}$ makes perovskites highly attractive for use in tandem solar cells, that is, as top cells of narrower-band gap absorbers such as silicon $(\mathrm{Si})$ and copper indium gallium selenide (CIGS). ${ }^{3-5}$

One of the main challenges in the perovskite field is the fabrication of high quality films with controlled morphology, high surface coverage, and minimum pinholes. ${ }^{6,7}$ Factors such as charge dissociation efficiency, charge transport, and diffusion length of charge species are strongly dependent on the film crystallinity. Therefore, the control of the crystallization and thus the kinetics of film formation during deposition and annealing are the key to boost the performance of solutionprocessed perovskite solar cells. ${ }^{8-11}$ For this purpose, approaches such as solvent engineering, ${ }^{12}$ inclusion of additives into the precursor solution, ${ }^{13-17}$ substrate temperature, ${ }^{18,19}$ thermal annealing, ${ }^{20}$ and solution-processed methods $^{21-23}$ have been investigated by several research groups.

Recently, it has been shown that improvements in crystallinity and larger grain sizes can impact the stability of the perovskite film ${ }^{19,24,25}$ and several solution-processed approaches to achieve micrometer-sized perovskite grains have been reported. ${ }^{18,19}$ Usually, these approaches involve hot-casting of the perovskite precursor solution onto a substrate maintained above the crystallization temperature of the perovskite phase (that is, between $145^{\circ} \mathrm{C}$ up to $180{ }^{\circ} \mathrm{C}$

Received: November 19, 2018

Accepted: February 25, 2019

Published: February 25, 2019 